\title{
Probabilistic Indicators of Structural Redundancy in Mechanics
}

\author{
Branko Blagojević ${ }^{1}$, Kalman Žiha ${ }^{2}$ \\ ${ }^{1}$ Department of Mechanical Engineering and Naval Architecture, Faculty of Electrical Engineering, Mechanical Engineering and \\ Naval Architecture (FESB), University of Split, Split, Croatia \\ ${ }^{2}$ Department of Ship Structures, Faculty of Mechanical Engineering and Naval Architecture (FSB), University of Zagreb, Zagreb, \\ Croatia \\ Email: bblag@fesb.hr,kziha@fsb.hr
}

Received July 20, 2012; revised August 23, 2012; accepted September 2, 2012

\begin{abstract}
The paper in the introductory part reviews various definitions and interpretations of structural redundancy in mechanics. The study focuses on the general structural redundancy of systems after sequences of component failures followed by possible load redistributions. The second section briefly summarizes the Event Oriented System Analysis and structural redundancy in terms of the conditional probabilistic entropy. Mechanical responses to adverse loads in this approach are represented by random operational and failure events in the lifetime. The general redundancy measure in the third section of the paper employs the information entropy and goes beyond existing formulations since it includes all functional modes in service. The paper continues with a summary of traditional redundancy indices. In addition, it proposes an alternative redundancy index that accounts for the transition to secondary functional level in case of failures of primary components. The example of a ship structure illustrates the usage of the conditional entropy of subsystems of operational events and compares it to the traditional and newly proposed redundancy indices. The study at the end investigates how to enhance the safety of structures by using the redundancy based design.
\end{abstract}

Keywords: Reliability; Event-Oriented System Analysis; Entropy; Redundancy; Redundancy Index; Structures

\section{Introduction}

Complex load-carrying engineering structures share a number of properties that relate to the overall structural safety. The definitions, applications and mathematical interpretations of important properties such as redundancy, robustness and vulnerability are still vague in different engineering disciplines [1-5]. Although those properties have been extensively investigated [6-9], the mechanical and structural redundancy has been one most difficult to quantify. It is usually classified into local and global (overall) redundancy and can be expressed in two forms, as the system reserve strength and the residual strength $[10,11]$. Reserve strength is the margin between the design load and the ultimate capacity of the overall structure to sustain the applied load.

The redundancy of complex engineering systems is commonly related to residual strength that remains in the structure after one or more components have failed [11, 12]. Descriptive redundancy assessment is characterized by a number of functional levels and by a number of alternative operational states. Mere deterministic measures, denoted as structural residual resistance factors and redundant factors which relate the strength of the intact and damaged structures, are considered as insufficient for problems of system redundancy.

The structural engineering for the sake of efficiency often focuses on "fail-safe" or "damage-tolerant" object that remain operational in the case of the first component failure. Redundancy in engineering systems in this paper is assessed as a general probabilistic operational abundance rather than deterministic survival ability in the case of first component failure. In complex structures with large number of interacting components possibly in different uncertain states, the redundancy becomes a significant issue in investigation of general system safety.

The study examines the benefits of combining the probabilistic reliability methods and the event oriented system analysis of structural redundancy using the same set of input data. EOSA relates the system redundancy to the conditional entropy of subsystems of failure events. Entropy as a measure of information primarily originates from the information theory $[13,14]$ and lately is generalized as an uncertainty measure in probability theory $[15,16]$. The unconditional information entropy has not been considered as yet as a useful measure of practical importance for structural systems in engineering.

However, the concept of conditional entropy in the 
probability theory represents an objective uncertainty measure for systems of events independent of anything else but possible events [17]. The redundancy defined by conditional entropy of failure path is found useful in assessment of lifeline systems efficiency in damaged conditions [18]. Therefore, the paper compares alternative definitions of the probabilistic redundancy indices to the entropy-based probabilistic redundancy measure in order to add impetus to further investigation of system safety enhancement in mechanical problems.

\section{Event Oriented System Analysis (EOSA)}

The event-oriented approach considers structures and structural components as systems and subsystems of random events in a lifetime. EOSA enumerates all observable, or at least the most important events, and designates their status as intact, operational, transitive, failure or collapsed. To every event status, a probability of occurrence can be assigned. By grouping events of different status, and associated probabilities, EOSA forms systems and subsystems of events. Jointly, the eventoriented system analysis combines modes analysis, enumeration of events, probability and information theory in order to quantify probabilistic systems' redundancy. Methods such as Secon Moment Methods (SM) First Order Reliability Methods (FOSM, FORM), Advanced FORM (AFORM), Second Order Reliability Methods (SORM) or Monte Carlo simulation and Bayesian methods are on disposal for probability calculations [19-23]. Most of these methods have been used in the assessment of safety of ship structures and structural components. EOSA uses these methods and provides additional information about structural behaviour through a calculation of entropies of systems of events. Additionally, methods of operational modes and effects analysis such as minimal cut-sets, minimal tie-sets, event-tree and fault-tree analysis can identify events $[24,25]$ and determine their relationships. EOSA methodology is summarized in the sequel.

A system, $S$, consists of all observable events $E_{k}$ with probabilities $p\left(E_{k}\right), k=1,2, \cdots, N$, where $N$ is the total number of events. Events can represent different functional states of the structure such as failure or operational state or some other state of the interest.

Event realization causes that system has a different functional status " $s$ ". EOSA designates events and systems' functional statuses with following indexes: $i$-intact (non-damaged), $c$-collapse, $t$-transitive, $n$-non-transitive, $o$-operational (with full or reduced capacity), $f$-failure, $d$-damage and combinations. Subsystems of events are made of the events of the same type. i.e. $S^{o}$ is then a designation of a subsystem of all operational events and $S^{f}$ is a subsystem of all failure events. Finite schemes are commonly used as mathematical presentation of the systems and subsystems of events [16]:

$$
S=\left(\begin{array}{ccc}
E_{1} & \cdots & E_{N} \\
p\left(E_{1}\right) & \cdots & p\left(E_{N}\right)
\end{array}\right)
$$

The systems of events can be also represented as a summa of subsystems of events:

$$
S=\left(S^{o}+S^{f}\right)
$$

$S^{o}$ ad $S^{f}$ are subsystems of operational and failure events

$$
\begin{gathered}
S^{o}=\left(\begin{array}{ccc}
E_{1}^{o} & \cdots & E_{N}^{o} \\
p\left(E_{1}^{o}\right) & \cdots & p\left(E_{N_{o}}^{o}\right)
\end{array}\right) \\
S^{f}=\left(\begin{array}{ccc}
E_{N_{o}+1}^{f} & \cdots & E_{N_{o}+N_{f}}^{f} \\
p\left(E_{N_{o}+1}^{f}\right) & \cdots & p\left(E_{N_{o}+N_{f}}^{f}\right.
\end{array}\right)
\end{gathered}
$$

$N=N_{o}+N_{f}$ is the total number of events in $S$.

The reliability of a system is equal to the probability of the occurrence of the subsystem of operational events:

$$
R(S)=p\left(S^{o}\right)=\sum_{k=1}^{N_{o}} p\left(E_{k}^{o}\right)
$$

The probability of failure of a system is equal to the probability of a failure subsystem:

$$
p_{f}(S)=p\left(S^{f}\right)=\sum_{k=N_{o}+1}^{N_{o}+N_{f}} p\left(E_{k}^{f}\right)
$$

For complete systems is $\Sigma p\left(E_{k}\right)=1$, otherwise $\Sigma p\left(E_{k}\right)$ $<1$.

Redundant structures are operational even if some of the structural components have failed. Thus, the redundant systems of events, representing redundant structures, have to be analysed as functional multi-level operational systems. A functional level can be understood as a system of events comprising all functional states of an object. The initial intact state of a structure is modelled as a system of events on the first functional level (primary level). After failure of one or more structural components, the system transits from the first level to the second level (secondary level). Further failures cause the system to transit to the third level, and so on.

An event ${ }^{l} E_{k}^{s}$ is of status " $s$ ", where $l=1,2, \cdots, n$ is a level and $k=1,2, \cdots,{ }^{l} N^{s}$, and ${ }^{l} N^{s}$ is the number of events of the same status " $s$ " within a functional level.

EOSA applies the entropy concept from information theory to assess the effects of the number of events, and dispersion of their probabilities, as well as the possible redistribution of loads after failures. The entropy in information theory is a logarithmic function that measures uncertainty originally introduced by [13]. The Rényi entropy is a generalization of Shannon entropy, and represents a family of functionals for quantifying the uncer- 
tainty of a system. The entropy of a system of events of order $\alpha$, for $\alpha \geq 0$ is [14]:

$$
H_{n}^{\alpha}(S)=\frac{1}{1-\alpha} \log _{2}\left(\sum_{i=1}^{n} p_{i}^{\alpha} / \sum_{i=1}^{n} p_{i}\right)
$$

where log base is commonly 2 . For the same probabilities of all events, Rényi entropy is $H_{n}^{\alpha}(S)=\log n$. Higher values of $\alpha$ give a Rényi entropy which is determined by events with the highest probability. Lower values of $\alpha$ give a Rényi entropy that weights events more equally, regardless of their probabilities. The case $\alpha$ $=1$ gives the Shannon entropy.

Equation (5) represents a measure of uncertainty corresponding to either incomplete or complete system of events. This is convenient for complex systems with large number of events where probabilities of some events cannot be determined, or even some events remain unknown.

System of events can also be considered under the condition that only the operational events are of interest. The conditional entropy of system $S$ can be obtained as follows [26]:

$$
H_{N_{o}}\left(S / S^{o}\right)=-\sum_{k=1}^{N_{o}} \frac{p\left(E_{k}^{o}\right)}{p\left(S^{o}\right)} \log \frac{p\left(E_{k}^{o}\right)}{p\left(S^{o}\right)}
$$

The entropy of the operational subsystem does not depend on the probability of a system $p(S)$. Nor does it depend on whether the system is complete or incomplete.

\section{The Probabilistic Redundancy Definition (Redundancy Assessment)}

The EOSA relates structural redundancy of the system $S$ to the uncertainty of the subsystem of operational modes $S^{o}[27]$ by employing the conditional entropy (6), as:

$$
R E D(S)=H_{N_{o}}\left(S / S^{o}\right)
$$

The events of a particular interest for redundant structures are the transitive events $E^{t}$ that cause the system ${ }^{l} S$ to transit from a current functional level $l$ to a subsequent level $l+1$ denoted ${ }^{l+1} S$. Every transitive event also leads to the emergence of a new functional state $j$ on the next level ${ }_{j}^{l+1} S^{s}$, of possibly different status such as operational, failure, collapse, transitive, etc. Functional states represent distinguished independent ways the object performs its functions with full or with reduced operational capacity. Newly emerged $j^{\text {th }}$ state at level $l+1$, after the transition event ${ }^{l} E_{k}^{t}$ occurred at level $l$, can be defined as a subsystem of compound secondary and primary transitive events ${ }_{j}^{l+1} S^{s} \cap{ }^{l} E_{k}^{t}$.

Conditional probability of transition from one level to the next level is defined as:

$$
p\left({ }_{j}^{l+1} S \cap{ }^{l} E_{k}^{t}\right)=p\left({ }_{j}^{l+1} S /{ }^{l} E_{k}^{t}\right) p\left({ }^{l} E_{k}^{t}\right)
$$

A practical measure of the probability of system's residual strength $p\left({ }^{1} S^{t}\right)$ on the primary level relates on one hand the probability of damage occurrence $p\left({ }^{1} S^{f}\right)$ with the probability of system collapse $p\left({ }^{1} S^{c}\right)$, and on the other, the probability of system operation $p\left({ }^{1} S^{o}\right)$ in undamaged condition with the probability of the intact system $p\left({ }^{1} S^{i}\right)$. The probability of the primary residual strength $p\left({ }^{1} S^{t}\right)$ equals then to the probability of the transitive modes and can be expressed as follows:

$$
p\left({ }^{1} S^{t}\right)=p\left({ }^{1} S^{f}\right)-p\left({ }^{1} S^{c}\right)=p\left({ }^{1} S^{o}\right)-p\left({ }^{1} S^{i}\right)
$$

The increase of the probability of residual strength, $p\left({ }^{1} S^{t}\right)$ in (9) practically can involve the diminution of the probability of the primary intact mode $p\left({ }^{1} S^{i}\right)$, rather than of the collapse mode $p\left({ }^{1} S^{c}\right)$, since $p\left({ }^{1} S^{i}\right) \gg p\left({ }^{1} S^{c}\right)$ for realistic objects. Therefore, the probability of residual strength for realistic objects has to be carefully selected in order to ensure the maximal probability of the primary intact mode $p\left({ }^{1} S^{i}\right)$, the minimal probability of the collapse mode $p\left({ }^{1} S^{c}\right)$ and the sufficient probability of transitive modes $p\left({ }^{1} S^{t}\right)$. Moreover, the subsequent functional states also have to satisfy the safety requirements imposed on a redundant system after next component failures.

The traditional probabilistic redundancy index $R_{I}$ is defined as the system's primary residual strength conditioned by any first component failure [28]. Such an index can be calculated in terms of the probabilities of primary structural level $l=1$ as shown:

$$
R_{I}=p\left({ }^{1} S^{t} /{ }^{1} S^{f}\right)=\frac{p\left({ }^{1} S^{t}\right)}{p\left({ }^{1} S^{f}\right)}
$$

The complement $R_{R}$ of the probabilistic redundancy index is defined as the system's primary collapse, conditioned by any first component failure [29] as shown:

$$
R_{R}=p\left({ }^{1} S^{c} /{ }^{1} S^{f}\right)=\frac{p\left({ }^{1} S^{c}\right)}{p\left({ }^{1} S^{f}\right)}=1-\frac{p\left({ }^{1} S^{t}\right)}{p\left({ }^{1} S^{f}\right)}=1-R_{I}
$$

The traditional redundancy index $R_{I}$ and its complement $R_{R}$ do not account for the intact mode.

The probabilistic redundancy factor $R_{F}$ can be defined [30], as the system's primary residual strength, conditioned by the collapse of the system, as follows:

$$
R_{F}=p\left({ }^{1} S^{t} /{ }^{1} S^{c}\right)=\frac{p\left({ }^{1} S^{t}\right)}{p\left({ }^{1} S^{c}\right)}=\frac{R_{I}}{1-R_{I}}
$$

Redundancy in engineering problems in this study is viewed rather as an operational abundance than mere survival ability after first component failures. Therefore, an alternative probabilistic redundancy index proposal is introduced in the study that considers the system's primary residual strength conditioned by operational mode 
as shown $[31,32]$.

$$
R_{O}=p\left({ }^{1} S^{t} /{ }^{1} S^{o}\right)=\frac{p\left({ }^{1} S^{t}\right)}{p\left({ }^{1} S^{o}\right)}=1-\frac{p\left({ }^{1} S^{i}\right)}{p\left({ }^{1} S^{o}\right)}
$$

The primary redundancy indices $(10,11,12$ and 13) do not account for the redistribution of loads in case of component failures. The probability of the reserve strength in case of load redistribution may be more appropriately expressed by the compound probabilities $p\left({ }_{j}^{2} S^{i} \cap{ }^{1} E_{k}^{t}\right)$ of the second level intact mode and first level transitive mode, denoted also as secondary reliability $p\left({ }^{2} S^{i}\right)$ calculated as:

$$
p\left({ }^{2} S^{i}\right)=\sum_{j=1}^{1} p\left({ }^{t} E_{j}^{t}\right) p\left({ }_{j}^{2} S^{i}\right)
$$

Moreover, the probability of the primary reserve strength may be viewed as the upper bound on the secondary reliability. This reasoning can be extended to a system with any number of functional levels representing redundant structure. The overall reliability of the multilevel system that accounts for probabilities of all intact modes on all levels $l=1, \cdots, n$ is calculated as follows:

$$
p\left(S^{o}\right)=p\left({ }^{n} S^{o}\right)=p\left({ }^{1} S^{i}\right)+p\left({ }^{2} S^{i}\right)+\cdots+p\left({ }^{n} S^{i}\right)
$$

\section{Example}

The example applies EOSA to investigate the probabilistic redundancy of a longitudinally stiffened structure located at the strength deck of a double-hull oil tanker (Figure 1).

The ship has following general characteristics: length between perpendiculars $174.8 \mathrm{~m}$; beam $31.4 \mathrm{~m}$; draught at full load $12.2 \mathrm{~m}$; block coefficient $C_{b}=0.82$; depth $17.5 \mathrm{~m}$; height of neutral axis from bottom $7552 \mathrm{~m}$; displacement (full load draught) 47,400 tons [33].

Structural analysis, done according to the DNV Rules $[34,35]$, showed that the considered structural configuration remained operational when some of the components have failed. All longitudinals and plating between longitudinals were involved in redundancy calculation as load carrying elements giving the total number of seven structural elements (Figure 1) on the first functional level $(l=1)$. After failure of one longitudinal stiffener the system transits into second functional level with six carrying elements on the second functional level $(l=2)$.

Finally, after failure of two longitudinal stiffeners the system transits into third functional level $(l=3)$ with five remain carrying elements $(3 \times$ plating, $\mathrm{T}$ girder and one remaining longitudinal stiffener).

First functional level includes the following random events: buckling of plating between longitudinals ( 3 basic events), torsion buckling of bulb longitudinals ( 3 basic events), yielding of bulb longitudinals (3 basic events), torsion buckling of $\mathrm{T}$ girder ( 1 basic event) and yielding of $\mathrm{T}$ girder ( 1 basic event). The first functional level has $n=11$ events designated ${ }^{l} A_{k}, k=1,2, \cdots, 11$.

Reliability indices are calculated according to [19]:

$$
{ }^{l} \beta_{i}=\frac{\mu_{l_{C_{i}}}-\mu_{l_{D_{i}}}}{\sqrt{\sigma^{2}{ }_{{ }_{l_{i}}}+\sigma^{2}{ }_{l_{D_{i}}}}}
$$

where $\mu_{l C_{i}}$ represent the mean values of stress random variables. In the cases of yield failure modes the mean values were taken as $60 \%$ of the material yield stress. In the cases of buckling failure modes the mean values were taken as calculated from the DNV formulas for critical buckling stress [35] for the corresponding structural element. $\mu_{l_{D_{i}}}$ represent mean values of load stresses determined in structural analysis. $\sigma_{l_{D_{i}}}$ and $\sigma_{l_{D_{i}}}$ represent corresponding standard deviations of the variables.

All the stress random variables are assumed to be independent and uncorrelated, with linear safety margins for all functional levels. All stress variable are taken to be log-normally distributed with coefficient of variation (COV) of 0.7 [36]. Reliabilities, $R$, and collapse probabilities, $p_{f}$, are calculated respectively:

$$
\begin{gathered}
R=p\left({ }^{1} A_{k}^{i}\right)=\Phi\left(-{ }^{1} \beta_{i}\right) \text { and } \\
p_{f}=p\left({ }^{1} A_{k}^{c}\right)=1-p\left({ }^{1} A_{k}^{i}\right)
\end{gathered}
$$

where $\Phi$ is the standard normal density function. For levels with only one state $j$ index is omitted for simplicity. The mean values of the wave induced bending moments and design pressure on deck are calculated according to the DNV Rules. The mean values of the still-water bending moments were taken as given in the trim and stability book for the full load state of the considered

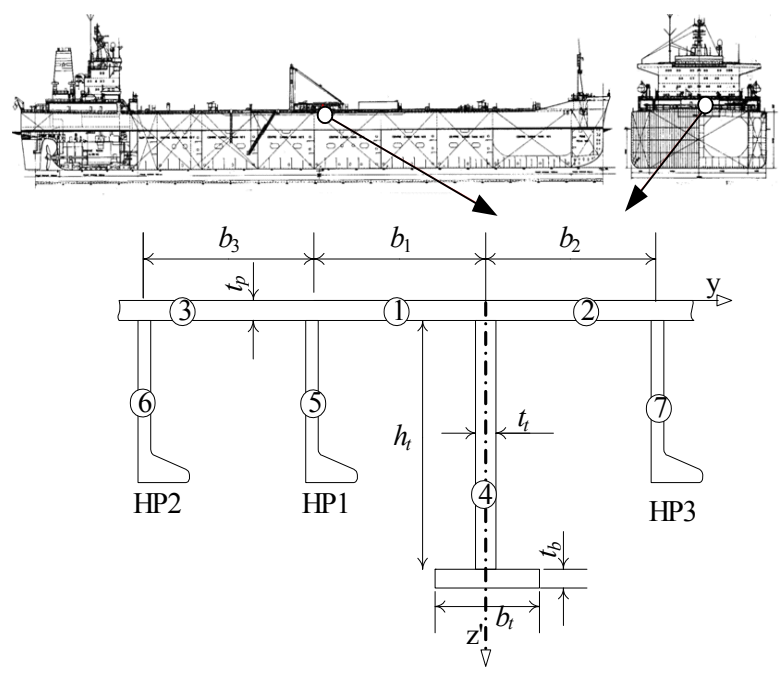

Figure 1. Location and configuration of the investigated panel of the tanker deck. 
ship. Statistical properties of random variables for tankers can be found in literature [37-39]. Selected data is presented in Tables 1 and 2 . In this investigation the following elements of the deck geometry were considered as deterministic variables: thickness of plating $t_{p}=14$ $\mathrm{mm}$, span of longitudinals $l=5.08 \mathrm{~m}$, spacing of longitudinals $s=0.8 \mathrm{~m}$, web height (T-profile) $h_{t}=450 \mathrm{~mm}$, web thickness (T-profile) $t_{t}=14 \mathrm{~mm}$, flange width (Tprofile) $b_{t}=100 \mathrm{~mm}$, flange thickness (T-profile) $t_{b}=14$ $\mathrm{mm}$, bulb longitudinals HP $220 \times 11.5$.

Table 3 shows properties of the tanker deck structure for all functional (operational) levels.

The number of compound events ${ }^{l} E_{1_{N}}$ on the first functional level is: ${ }^{1} N=2^{n}=2^{11}=2048$. There is only one intact functional state represented by the event ${ }^{1} E_{1}^{i}$. Collapse of either one of the longitudinals HP2 or HP3 (Figure 1) causes transition from the first functional level to the second functional level. There is ${ }^{1} N^{t}=15$ transitive events on the first level, denoted ${ }^{1} E_{k}^{t}, k=1,2$, $\cdots,{ }^{1} N^{t}$. The remaining ${ }^{1} N^{\mathrm{c}}=2032$ events on the first level represent collapse of the structure.

Table 1. Statistical properties of material and loads.

\begin{tabular}{cccc}
\hline & Mean & Distribution & COV \\
\hline $\begin{array}{c}\text { Yield stress (mild } \\
\text { shipbuilding steel) }\end{array}$ & $235 \mathrm{~N} / \mathrm{mm}^{2}$ & Log-normal & 0.06 \\
Modulus of elasticity & $206,000 \mathrm{~N} / \mathrm{mm}^{2}$ & Normal & 0.01 \\
$\begin{array}{c}\text { Stillwater bending } \\
\text { moment (sagging) }\end{array}$ & $296,252 \mathrm{kNm}$ & Normal & 0.4 \\
$\begin{array}{c}\text { Stillwater bending } \\
\text { moment (hogging) }\end{array}$ & $244,690 \mathrm{kNm}$ & Normal & 0.4 \\
$\begin{array}{c}\text { Wave-induced bending } \\
\text { moment (sagging) }\end{array}$ & $1,533,336 \mathrm{kNm}$ & Gumbel & 0.09 \\
$\begin{array}{c}\text { Wave-induced bending } \\
\text { moment (hogging) }\end{array}$ & $1,428,791 \mathrm{kNm}$ & Gumbel & 0.09 \\
$\begin{array}{c}\text { Design pressure (deck) } \\
\text { Mesignal }\end{array}$ & $13.6 \mathrm{kN} / \mathrm{m}^{2}$ & Normal & 0.09 \\
\hline
\end{tabular}

Table 2. Statistical properties of the deck panel.

\begin{tabular}{cccc}
\hline & Mean & Distribution & COV \\
\hline Width of effective plate flange & $800 \mathrm{~mm}$ & Normal & 0.01 \\
$\begin{array}{c}\text { Section modulus (longitudinals } \\
\text { and plate flange } b_{\mathrm{i}} \text { ) }\end{array}$ & $326 \mathrm{~cm}^{3}$ & Log-normal & 0.04 \\
$\begin{array}{c}\text { Midship section modulus at deck } \\
\text { M }\end{array}$ & $16.14 \mathrm{~m}^{3}$ & Log-normal & 0.04 \\
\hline
\end{tabular}

Table 3. Properties of the deck structure (all levels).

\begin{tabular}{cccc}
\hline & $\begin{array}{c}\text { Level } \\
\boldsymbol{L}=\mathbf{1}\end{array}$ & $\begin{array}{c}\text { Level } \\
\boldsymbol{L}=\mathbf{2}\end{array}$ & $\begin{array}{c}\text { Level } \\
\boldsymbol{L}=\mathbf{3}\end{array}$ \\
\hline Panel cross sectional area in $\mathrm{cm}^{2}$ & 392 & 360 & 327 \\
Panel neutral axis in cm & 9.3 & 8.9 & 8.4 \\
Panel moment of inertia in $\mathrm{cm}^{4}$ & 74,021 & 71,021 & 67,785 \\
\hline
\end{tabular}

Events are appropriately grouped according to their functional state and the structure is modelled by the system of events with three subsystems: intact, transitive and collapse. The system can be written as

$$
{ }^{1} S=\left({ }^{1} S^{i}+{ }^{1} S^{t}+{ }^{1} S^{c}\right) .
$$

The probability of intact state on the first functional level is calculated as:

$$
p\left({ }^{1} E_{1}^{i}\right)=p\left({ }^{1} S^{i}\right)=\prod_{k=1}^{11} p\left({ }^{1} A_{k}^{i}\right)=0.9751
$$

The probabilities of transitive events at the first level can be calculated likewise:

$$
\begin{aligned}
p\left({ }^{1} E_{1}^{t}\right) & =\prod_{k=1}^{6} p\left({ }^{1} A_{k}^{i}\right) \cdot p\left({ }^{1} A_{7}^{c}\right) \cdot \prod_{k=8}^{11} p\left({ }^{1} A_{k}^{i}\right)=0.1853 \times 10^{-3} \\
p\left({ }^{1} E_{2}^{t}\right) & =\prod_{k=1}^{7} p\left({ }^{1} A_{k}^{i}\right) \cdot p\left({ }^{1} A_{8}^{c}\right) \cdot \prod_{k=9}^{11} p\left({ }^{1} A_{k}^{i}\right)=0.1853 \times 10^{-3} \\
p\left({ }^{1} E_{3}^{t}\right) & =\prod_{k=1}^{9} p\left({ }^{1} A_{k}^{i}\right) \cdot p\left({ }^{1} A_{10}^{c}\right) \cdot p\left({ }^{1} A_{11}^{i}\right)=0.5985 \times 10^{-2}
\end{aligned}
$$

The probabilities of the remaining events are:

$$
\begin{gathered}
p\left({ }^{1} E_{4}^{t}\right)=0.5985 \times 10^{-2}, \quad p\left({ }^{1} E_{5}^{t}\right)=0.3522 \times 10^{-7}, \\
p\left({ }^{1} E_{6}^{t}\right)=0.1137 \times 10^{-5}, \quad p\left({ }^{1} E_{7}^{t}\right)=0.1137 \times 10^{-5}, \\
p\left({ }^{1} E_{8}^{t}\right)=0.1137 \times 10^{-5}, \quad p\left({ }^{1} E_{9}^{t}\right)=0.1137 \times 10^{-5}, \\
p\left({ }^{1} E_{10}^{t}\right)=0.3673 \times 10^{-4}, \quad p\left({ }^{1} E_{11}^{t}\right)=0.2162 \times 10^{-9}, \\
p\left({ }^{1} E_{12}^{t}\right)=0.2162 \times 10^{-9}, \quad p\left({ }^{1} E_{13}^{t}\right)=0.6981 \times 10^{-8}, \\
p\left({ }^{1} E_{14}^{t}\right)=0.6981 \times 10^{-8}, \quad p\left({ }^{1} E_{15}^{t}\right)=0.1327 \times 10^{-11}
\end{gathered}
$$

The subsystem of transitive events is modelled as:

$$
{ }^{1} S^{t}=\left(\begin{array}{cccc}
{ }^{1} E_{1}^{t} & { }^{1} E_{2}^{t} & \cdots & { }^{1} E_{15}^{t} \\
p\left({ }^{1} E_{1}^{t}\right) & p\left({ }^{1} E_{2}^{t}\right) & \cdots & p\left({ }^{1} E_{15}^{t}\right)
\end{array}\right)
$$

Probability of the subsystem of transitive events represents the probability of transition from the primary to the secondary level also denoted as the probability of the primary residual strength and can be calculated as:

$$
p\left({ }^{1} S^{t}\right)=\sum_{k=1}^{15} p\left({ }^{1} E_{k}^{t}\right)=0.0124
$$

The subsystem of collapse events is modelled as:

$$
{ }^{1} S^{c}=\left(\begin{array}{ccc}
{ }^{1} E_{1}^{c} & \cdots & { }^{1} E_{2032}^{c} \\
p\left({ }^{1} E_{1}^{c}\right) & \cdots & p\left({ }^{1} E_{2032}^{c}\right)
\end{array}\right)
$$

The probabilities of individual collapse events are not listed here due to large number of events $\left(N_{c}=2032\right)$. Probability of the subsystem of collapse events is: 


$$
p\left({ }^{1} S^{c}\right)=\sum_{k=1}^{2032} p\left({ }^{1} E_{k}^{c}\right)=0.0125
$$

One can easily check that on the first functional level the following relation among probabilities holds:

$$
\begin{aligned}
& p\left({ }^{1} S^{i}\right)+p\left({ }^{1} S^{t}\right)+p\left({ }^{1} S^{c}\right) \\
& =0.9751+0.0124+0.0125=1
\end{aligned}
$$

The second functional level emerges when one of the longitudinals, HP2 or HP3, collapses. The remaining part of a structure is still operational, but now with reduced load-carrying capacity. In this case, it is assumed that collapsed longitudinal has no remaining load-carrying capacity.

The load is then redistributed to the remaining elements on the deck structure and new values of reliability and probability of failure are calculated.

When any of the longitudinals fails the structural configuration reduces to 6 load-carrying elements: $3 \times$ deck plating, $\mathrm{T}$ longitudinal and remaining two HP longitudinals.

Hence, the system of events on the second functional level has 9 basic events with probabilities calculated in a same way as for the first functional level:

$$
\begin{aligned}
& p\left({ }^{2} A_{1}^{i}\right)=0.9955, \quad p\left({ }^{2} A_{2}^{i}\right)=0.9955, \quad p\left({ }^{2} A_{3}^{i}\right)=0.9955, \\
& p\left({ }^{2} A_{4}^{i}\right)=0.9999, \quad p\left({ }^{2} A_{5}^{i}\right)=0.9960, \quad p\left({ }^{2} A_{6}^{i}\right)=0.9999 \\
& p\left({ }^{2} A_{7}^{i}\right)=0.9999, \quad p\left({ }^{2} A_{8}^{i}\right)=0.9855, \quad p\left({ }^{2} A_{9}^{i}\right)=0.9855 .
\end{aligned}
$$

Probability of occurrence of one compound intact event on the second functional level is:

$$
p\left({ }^{2} E_{1}^{i}\right)=\prod_{k=1}^{9} p\left({ }^{2} A_{k}^{i}\right)=0.9541
$$

System and subsystems of events, representing deck structure, are presented on Figure 2. The diagram shows levels, subsystems and events as well as transition paths from one functional level to another. This figure illustrates complexity of modelling of multi-level operational redundant structures.

However, a number of random events have extremely low probabilities of occurrence, which gives the opportunity to reduce the number of events in future development of the EOSA methodology.

Failure of the remaining longitudinal, HP2 or HP3, causes emergence of the third functional level. Thus, the second functional level also includes three subsystems: intact, collapse and transitive. The second level is modelled by the following system of events:

$$
{ }^{2} S=\left({ }^{1} S^{i},{ }_{1}^{2} S \cap{ }^{1} E_{1}^{t}, \cdots,{ }_{j}^{2} S \cap{ }^{1} E_{j}^{t}, \cdots,{ }_{1} N^{t} S \cap{ }^{2} E_{{ }_{1} N^{t}}^{t},{ }^{1} S^{c}\right)
$$

This level consist of non-transitive events from the first level (intact, collapse) and new states that emerge $\left({ }_{1}^{2} S \cap{ }^{1} E_{1}^{t}, \cdots,{ }_{15}^{2} S \cap{ }^{1} E_{15}^{t}\right)$ due to occurrence of some transitive event on the previous level. From 15 transitive events on the first level 15 functional states will emerge on the second level. However, only six of those new states are further transitive (Figure 2). Every transitive state on level 2 has 3 transitive events causing emergence of 18 new states on the third level (Figure 2). The probabilities of occurrence of transitive events for the first functional state $j=1$ on the second level are calculated:

$$
\begin{aligned}
p\left({ }^{2} E_{1}^{t}\right) & =\prod_{k=1}^{6} p\left({ }^{2} A_{k}^{i}\right) \cdot p\left({ }^{2} A_{7}^{c}\right) p\left({ }^{2} A_{8}^{i}\right) p\left({ }^{2} A_{9}^{i}\right) \\
& =0.9555 \times 10^{-5} \\
p\left({ }^{2} E_{2}^{t}\right) & =\prod_{k=1}^{8} p\left({ }^{2} A_{k}^{i}\right) p \cdot\left({ }^{2} A_{9}^{c}\right)=0.1407 \times 10^{-1} \\
p\left({ }^{2} E_{3}^{t}\right) & =\prod_{k=1}^{6} p\left({ }^{2} A_{k}^{i}\right) \cdot p\left({ }^{2} A_{7}^{c}\right) p\left({ }^{2} A_{8}^{i}\right) p\left({ }^{2} A_{9}^{c}\right) \\
& =0.1408 \times 10^{-6}
\end{aligned}
$$

Probabilities of the remaining transitive events and collapse events can be calculated in a same way. Since the total number of compound events on the second level is ${ }^{2} N=9713$ the probabilities of remaining events are not listed here. The reliability $p\left({ }^{2} S^{i}\right)$ and the probability of failure $p\left({ }^{2} S^{c}\right)$ for the second functional level are:

$$
\begin{aligned}
p\left({ }^{2} S^{i}\right) & =\sum_{k=1}^{{ }^{1}} p\left({ }^{1} E_{k}^{t}\right) \sum_{k=1}^{2} p\left({ }^{2} E_{k}^{i}\right)=\sum_{j=1}^{1} p\left({ }^{t} E_{j}^{t}\right) p\left({ }_{j}^{2} S^{i}\right) \\
& =0.0118 \\
p\left({ }^{2} S^{c}\right) & =\sum_{k=1}^{{ }^{1} N^{t}} p\left({ }^{1} E_{k}^{t}\right) \sum_{k=1}^{2} p\left({ }^{N^{c}} E_{k}^{c}\right)=\sum_{j=1}^{{ }^{1}} p\left({ }^{t} E_{j}^{t}\right) p\left({ }_{j}^{2} S^{c}\right) \\
& =5.6 \times 10^{-4}
\end{aligned}
$$

The overall reliability of the system with 2 functional levels, $p\left({ }^{2} S^{o}\right)$, is probability that transition from the first level to the second level will result in occurrence of event representing one of undamaged states of second level. It includes all probabilities of intact states on the first level as well as all compound intact states on the second level of transitive states on the first level $\left({ }^{1} E_{k}^{t}\right)$ and one intact event on the second level $\left({ }^{2} E_{1}^{i}\right)$ :

$$
p\left({ }^{2} S^{o}\right)=p\left({ }^{1} S^{i}\right)+p\left({ }^{2} S^{i}\right)=0.9869
$$

Adequately, the overall probability of failure is then:

$$
\begin{aligned}
& p\left({ }^{2} S^{f}\right)=p\left({ }^{1} S^{c}\right)+p\left({ }^{2} S^{c}\right) \\
& =0.0125+5.689 \times 10^{-4}=0.0131
\end{aligned}
$$

Again, $p\left({ }^{2} S^{o}\right)+p\left({ }^{2} S^{f}\right)=1$ and the second level system $\left({ }^{2} S\right)$ is complete system of events.

The third functional level $(l=3)$ arises when failure of both longitudinals, HP2 and HP3, occurs (not necessarily simultaneously). On the third level there is 5 structural 


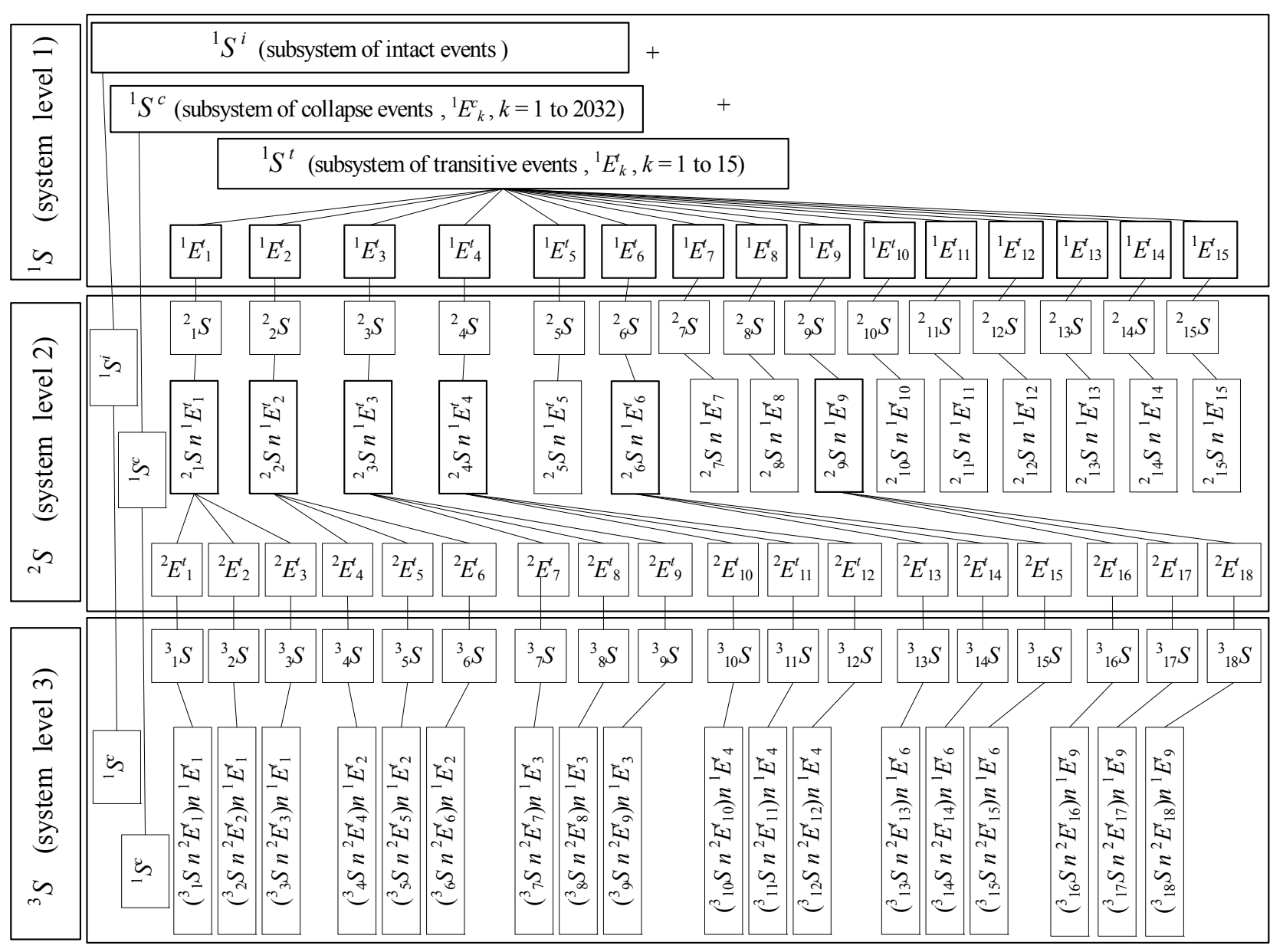

Figure 2. EOSA model of multi-level system of events representing part of tanker structure.

elements remained to carry redistributed load: $3 \times$ plating, $\mathrm{T}$ girder and longitudinal HP1. This structural configuration represents non-redundant structure, since further damage of any element will cause the entire structure to collapse. The number of basic events on the third level is connected to the number of possible types of failure of the remaining structural elements, thus giving 7 basic events ${ }^{3} n=7$.

There is 18 operational states on the third level emerging from $j=1,2, \cdots, 18$ compound transitive events on the second functional level (Figure 2). Each one of the 18 operational states on the third level has one intact event. All the remaining events are collapse events, i.e. there are no transitive events on the third level (non-redundant structure).

The total number of events on the third level is ${ }^{3} N=$ 12017. The probabilities of 7 basic events are:

$$
\begin{aligned}
& p\left({ }^{3} A_{1}^{i}\right)=0.9874, p\left({ }^{3} A_{2}^{i}\right)=0.9874, p\left({ }^{3} A_{3}^{i}\right)=0.9874 \\
& p\left({ }^{3} A_{4}^{i}\right)=0.9999, p\left({ }^{3} A_{5}^{i}\right)=0.9887, p\left({ }^{3} A_{6}^{i}\right)=0.9999 \\
& p\left({ }^{3} A_{7}^{i}\right)=0.9660
\end{aligned}
$$

Probabilities of all intact states are equal:

$$
p\left({ }_{j}^{3} S^{i}\right)=\prod_{k=1}^{7} p\left({ }^{3} A_{k}^{i}\right)=0.9195, j=1,2, \cdots, 18 .
$$

Probabilities of 18 collapse states are:

$$
p\left({ }_{j}^{3} S^{c}\right)=0.0804, j=1,2, \cdots, 18 .
$$

The third level is modelled as the system of events consisting of the non-transitive events on the second level together with the new states on the third level (compound events). Compound probabilities on the third level are equal to the probabilities of the transitive events on the previous levels:

$$
p\left[\left({ }_{j}^{3} S \cap{ }^{2} E_{j}^{t}\right) \cap{ }^{1} E_{k}^{t}\right]=p\left({ }_{j}^{3} S\right) p\left({ }^{2} E_{j}^{t}\right) p\left({ }^{1} E_{k}^{t}\right)
$$

Reliabilities $p\left({ }^{3} S^{i}\right)$ and probabilities of failure $p\left({ }_{j}^{3} S^{c}\right)=$ are calculated, using the same approach as before, by means of a computer:

$$
p\left({ }^{3} S^{i}\right)=1.5977 \times 10^{-4} \text { and } p\left({ }^{3} S^{c}\right)=1.3979 \times 10^{-5}
$$

The third functional level can be also viewed as a system of subsystems ${ }^{3} S=\left({ }^{3} S^{o}+{ }^{3} S^{f}\right)$, where the subsys- 
tem of operational states is ${ }^{3} S^{o}=\left({ }^{1} S^{i}+{ }^{2} S^{i}+{ }^{3} S^{i}\right)$ and subsystem of failure states is ${ }^{3} S^{f}=\left({ }^{1} S^{c}+{ }^{2} S^{c}+{ }^{3} S^{c}\right)$.

The overall reliability includes all probabilities of intact states of the first level and the probabilities of transitive and intact states on the second and the third level:

$$
p\left({ }^{3} S^{o}\right)=p\left({ }^{1} S^{i}\right)+p\left({ }^{2} S^{i}\right)+p\left({ }^{3} S^{i}\right)=0.9870
$$

The total probability of collapse includes all probabilities of collapse states on the first level as well as compound probabilities of transitive and collapse states on the second and third level:

$$
p\left({ }^{3} S^{f}\right)=p\left({ }^{1} S^{c}\right)+p\left({ }^{2} S^{c}\right)+p\left({ }^{3} S^{c}\right)=0.0130
$$

Since $p\left({ }^{3} S^{o}\right)+p\left({ }^{3} S^{f}\right)=0.9870+0.0130=1$, the system of events that models the structure is complete.

The maximum entropy on the first functional level is $\log \left({ }^{1} N\right)=\log (2048)=11.0$. The conditional entropy of the first functional level with respect to the operational modes denoted as redundancy with respect to the operational mode is according to Equations (5) and (6):

$$
H\left({ }^{1} S /{ }^{1} S^{o}\right)=\operatorname{RED}\left({ }^{1} S^{o}\right)=0.1125 \text { bits. }
$$

The conditional entropy of the system of events for the second level is then:

$$
H\left({ }^{2} S /{ }^{2} S^{i}\right)=R E D\left({ }^{2} S^{i}\right)=1.0245 \text { bits. }
$$

System redundancy after inclusion of the third level is:

$$
\operatorname{RED}\left({ }^{3} S^{i}\right)=H\left({ }^{3} S /{ }^{3} S^{i}\right)=1.1971 \text { bits. }
$$

Maximum redundancy is $\operatorname{RED}\left({ }^{3} S^{i}\right)_{\max }=4.1699$ bits.

The first aim of the redundancy based design in the example was to determine the spacing of deck longitudinal that lead to the most redundant deck topology, that is, with largest $R E D(S)$, starting from the prototype spacing of $80 \mathrm{~cm}$. The parametric study of structural redundancy was performed for the range of spacing of deck longitudinal between 63 and $97 \mathrm{~cm}$ with following constraints:

- The target reliability of a modified structure is equal or larger than the reliability of the initial structure.

- The weight of a modified structure is constant.

The analysis resulted with the highest redundancy $R E D(S)=1.7769$ for configuration with $b_{1}=69 \mathrm{~cm}, b_{2}=$ $80 \mathrm{~cm}$ and $b_{3}=91 \mathrm{~cm}$ (Figure 3), which significantly differs to the prototype $\left(b_{1}=b_{2}=b_{3}=80 \mathrm{~cm}\right)$. The reliability of that configuration is $R=0.981$. The highest redundancy indicates the structural configuration with the most uniformly distributed probabilities of operational modes for each structural element.

The second aim of the redundancy based design in the study is to compare the entropy based redundancy measure $R E D(S)$ to the newly proposed probabilistic reliability index $R_{\mathrm{o}}$ and to the traditional redundancy index $R_{i}$, Figure 4. The three redundancy measures are computa-

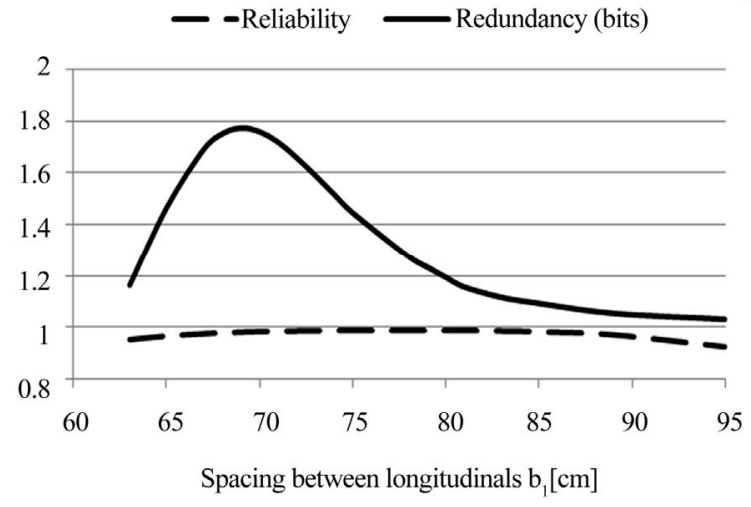

Figure 3. Results of redundancy based design of the part of ship deck structure.

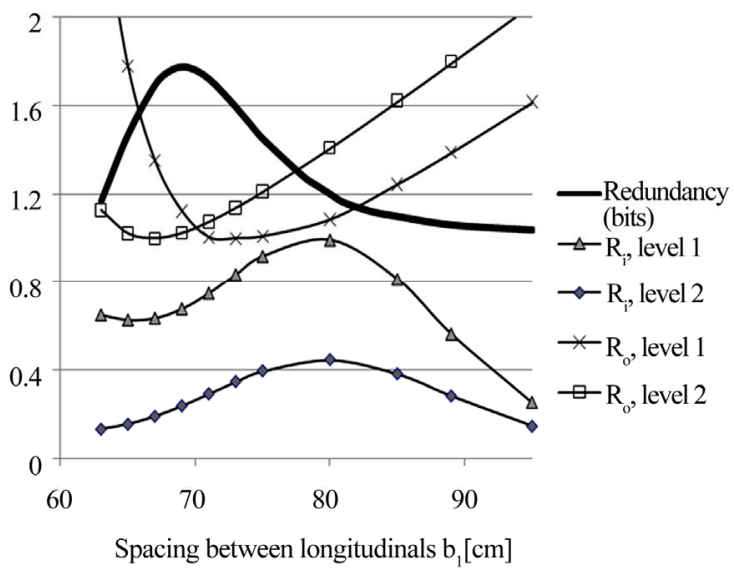

Figure 4. Redundancy indices $R_{i}$ and $R_{o}$ for the first two functional levels to the entropy based redundancy measure.

tionally not compliant due to different methodologies in their definitions. Values of $R_{o}$ indices in the example are normalized with respect to the minimum gained values of $R_{o}$ at each level. The entropy based redundancy measure $R E D(S)$ depends on the probability of operational modes at all functional levels and the values of redundancy indices $R_{o}$ and $R_{\mathrm{i}}$ depend on the probabilities of transitional subsystems on the first level only.

The maximum of the traditional index $R_{\mathrm{i}}$ indicates a different structural configuration as the best, for $b_{1}=79$ $\mathrm{cm}, b_{2}=80 \mathrm{~cm}, b_{3}=81 \mathrm{~cm}$, on both levels. However, the maximum of $R_{o}$ redundancy index shows a good trend as its values strive towards configuration with maximum redundancy in each new level. On the first level the best index value is gained for $b_{1}=74 \mathrm{~cm}, b_{2}=80 \mathrm{~cm}, b_{3}=86$ $\mathrm{cm}$ on the second level best index value is gained for $b_{1}=$ $67 \mathrm{~cm}, b_{2}=80 \mathrm{~cm}, b_{3}=93 \mathrm{~cm}$ that almost coincides with the values of the configuration with highest $R E D(S)$.

\section{Conclusions}

Most of the engineering decisions are governed by con- 
siderations about matter and energy in the physical space. Some of the decisions are additionally supported by the probabilistic and statistical laws governing the probability space. However, the event-oriented system analysis (EOSA) combines the physical and the probabilistic space to facilitate some of the engineering decisions using the entropy concept valid in the event space.

The paper compared the entropy-based redundancy measure and the probabilistic redundancy indices attainable within the EOSA. The underlying idea of the study is that the entropy-based redundancy measure most appropriately captures the intuited meaning of the structural redundancy even in case of cascades of failures and load redistribution. The example reveals at this point of the investigation that the newly proposed redundancy index $R_{o}$ in the paper, which relates the primary residual strength to the operational modes only for the first component failure, can be used as an indicator that approximates structural redundancy. It follows at the primary level that the trend of the entropy-based redundancy measure $R E D(S)$ that accounts for the multilevel sequences of component failures more appropriately of the traditional redundancy index.

The advantage of the redundancy index, where applicable, over the entropy-based redundancy measure, is in simplicity and lower calculation effort. However, for more precise conclusion of redundancy of complex, multilevel operational structures with a large number of individual components, the calculations of all functional levels are necessary. The paper acknowledges that the eventoriented analysis of redundant objects exposed to successive component failures, which change the system configuration and provoke a redistribution of demands and capabilities, is a complicated task, but it also proves that it is feasible attainable by appropriate software.

Finally, the study confirms that the EOSA has potentialities for structural safety enhancement by adopting the entropy concept in the maximal redundancy principle for the redundancy-based design.

\section{REFERENCES}

[1] M. Arvidsson and I. Gremyr, "Principles of Robust Design Methodology," Quality and Reliability Engineering International, Vol. 24, No. 1, 2008, pp. 24-35.

[2] G. Taguchi, S. Chowdhury and S. Taguchi, "Robust Engineering-Learn How to Boost Quality While Reducing Costs and Time to Market," McGraw-Hill, New York, 2000 .

[3] J. Agarwal, D. I. Blockley and N. J. Woodman, "Vulnerability of structural systems," Structural Safety, Vol. 25, No. 3, 2003, pp. 263-286. doi:10.1016/S0167-4730(02)00068-1

[4] A. Eriksson and A. G. Tibert, "Redundant and ForceDifferentiated Systems in Engineering and Nature," Com- puter Methods in Applied Mechanics and Engineering, Vol. 195, No. 41, 2006, pp. 5437-5453.

doi:10.1016/i.cma.2005.11.007

[5] J. W. Baker, M. Schubert and M. H. Faber, "On the Assessment of Robustness," Structural Safety, Vol. 30, No. 3, 2008, pp. 253-267. doi:10.1016/j.strusafe.2006.11.004

[6] B. W. Schafer and P. Bajpai, "Stability Degradation and Redundancy in Damaged Structures," Engineering Structures, Vol. 27, No. 11, 2005, pp. 1642-1651. doi:10.1016/j.engstruct.2005.05.012

[7] L. Dueñas-Osorio and S. M. Vemuru, "Cascading Failures in Complex Infrastructure Systems," Structural Safety, Vol. 31, No. 2, 2009, pp. 157-167. doi:10.1016/j.strusafe.2008.06.007

[8] Z. Tian and M. J. Zuo, "Redundancy Allocation for Multi-State Systems Using Physical Programming and Genetic Algorithms," Reliability Engineering and System Safety, Vol. 91, No. 9, 2006, pp. 1049-1056. doi:10.1016/i.ress.2005.11.039

[9] J. England, J. Agarwal and D. Blockley, "The Vulnerability of Structures to Unforeseen Events," Computers \& Structures, Vol. 86, No. 10, 2008, pp. 1042-1051. doi:10.1016/j.compstruc.2007.05.039

[10] S. G. Stiansen, "Interrelation between Design, Inspection and Redundancy in Marine Structures," National Academy Press, Washington DC, 1984.

[11] Y. Feng, "The Theory of Structural Redundancy and Its Effect on Structural Design," Computers \& Structures, Vol. 28, No. 1, 1988, pp. 15-24. doi:10.1016/0045-7949(88)90087-9

[12] K. Chen and S. Zhang, "Semi-Probabilistic Method for Evaluating Systems Redundancy of Existing Offshore Structures," Ocean Engineering, Vol. 23, No. 6, 1996, pp. 455-464. doi:10.1016/0029-8018(95)00051-8

[13] C. E. Shannon and W. Weaver, "The Mathematical Theory of Communication," Urbana University of Illinois Press, Urbana, 1949.

[14] A. Rényi, "Probability Theory," North-Holland, Amsterdam, 1970.

[15] J. Aczel and Z. Daroczy, "On Measures of Information and Their Characterization," Academic Press, New York, 1975.

[16] A. I. Khinchin, "Mathematical Foundations of Information Theory," Dover Publications, New York, 1957.

[17] K. Žiha, "Event Oriented System Analysis," Probabilistic Engineering Mechanics, Vol. 15, No. 3, 2000, pp. 261275. doi:10.1016/S0266-8920(99)00025-9

[18] M. Hoshiya, K. Yamamoto and H. Ohno, "Redundancy Index of Lifelines for Mitigation Measures against Seismic Risk," Probabilistic Engineering Mechanics, Vol. 19, No. 3, 2004, pp. 205-210.

doi:10.1016/j.probengmech.2004.02.003

[19] H. Madsen, S. Krenk and N. C. Lind, "Methods of Structural Safety," Prentice Hall, Englewood Cliffs, 1986.

[20] A. H. S. Ang and W. H. Tang, "Probability Concepts in Engineering," John Wiley, New York, 2007.

[21] S. K. Choi, R. V. Grandhi and R. A. Canfield, "Reliabil- 
ity-Based Structural Design,” Springer, New York, 2007.

[22] M. Rausand and A. Høyland, "System Reliability Theory: Models, Statistical Methods, and Applications," WileyInterscience, New York, 2003.

[23] R. Y. Rubinstein and D. P. Kroese, "Simulation and the Monte Carlo Method," Wiley-Interscience, New York, 2007. doi:10.1002/9780470230381

[24] M. S. Hamada, A. Wilson, C. S. Reese and H. F. Martz, "Bayesian Reliability," Springer, New York, 2008. doi:10.1007/978-0-387-77950-8

[25] D. L. Kreher and D. R. Stinson, "Combinatorial Algorithms: Generation, Enumeration, and Search," CRC Press, Boca Raton, 1998.

[26] K. Žiha, "Event-Oriented Analysis of Series Structural Systems," Structural Safety, Vol. 23, No. 1, 2001, pp 129. doi:10.1016/S0167-4730(00)00022-9

[27] K. Žiha, "Redundancy and Robustness of Systems of Events," Probabilistic Engineering Mechanics, Vol. 15, No. 4, 2000, pp. 347-357. doi:10.1016/S0266-8920(99)00036-3

[28] C. Palion, M. Shinozuka and Y. N. Chen, "Reliability Analysis of Offshore Structures," Marine Structural Reliability Symposium, New York, 1987.

[29] R. S. De, A. Karamchandani and C. A. Cornell, "Study of Redundancy in Near-Ideal Parallel Structural Systems," Structural Safety and Reliability, Vol. 2, 1990, pp. 975982.

[30] S. Hendawi and D. M. Frangopol, "System Reliability and Redundancy in Structural Design and Evaluation," Structural Safety, Vol. 16, No. 1-2, 1994, pp. 47-71. doi:10.1016/0167-4730(94)00027-N
[31] K. Žiha, "Redundancy Based Design by Event Oriented Analysis," Transactions of FAMENA, Vol. 27, No. 2, 2003, pp. 1-12.

[32] K. Žiha, "Event-Oriented Analysis of Fail-Safe Objects," Transactions of FAMENA, Vol. 27, No. 1, 2003, pp. 1122.

[33] B. Blagojević and K. Žiha, "On the Assessment of Redundancy of Ship Structural Components," Proceedings of the ASME 27th International Conference on Offshore Mechanics and Arctic Engineering OMAE 2008, Estoril, 15-20 June 2008, pp. 256-262.

[34] N. Veritas, "Ship's Load and Strength Manual," Det Norske Veritas, Oslo, 1995.

[35] N. Veritas, "Rules for Classification of Ships," Det Norske Veritas, Oslo, 2007.

[36] I. A. Assakkaf, "Reliability-Design of Panels and Fatigue Details of Ship Structures," University of Maryland, College Park, 1998.

[37] C. G. Soares and P. M. Dogliani, "Probabilistic Modelling of Time-Varying Still-Water Load Effects in Tankers," Marine Structures, Vol. 13, No. 2, 2000, pp. 129143. doi:10.1016/S0951-8339(00)00006-X

[38] K. Atua, I. A. Assakkaf and M. Ayyub, "Statistical Characteristics of Strength and Load Random Variable of Ship Structures," Proceedings of the 7th Specialty Conference on Probabilistic Mechanisms and Structural Reliability, Worcester, 7-9 August 1996.

[39] J. Parunov, I. Senjanović and C. G. Soares, "Hull-Girder Reliability of New Generation Oil Tanker," Marine Structures, Vol. 20, No. 1-2, 2007, pp. 49-70. 